\title{
Complutum
}

ISSN: 1131-6993

\section{El techo de mi casa es particular: identidades étnicas y espacios domésticos en el distrito de Bunkpurugu-Yunyoo (noreste de Ghana) ${ }^{1}$}

\author{
Manuel Calvo Trias ${ }^{2 a}$; Jaume García Rossellób; David Javaloyas Molina ${ }^{2 c}$; Daniel Albero Santacreu²
}

Recibido: 10 de julio de 2016 / Aceptado: 5 de marzo de 2017

Resumen. En el presente artículo tratamos de entender las profundas y complejas relaciones que se establecen entre las identidades étnicas y la cultura material. Para ello, hemos desarrollado un estudio etnoarqueológico de los espacios domésticos de diversas comunidades que habitan en el distrito de Bunkpurugu-Yunyoo (Noreste de Ghana). Este territorio está habitado por diferentes grupos étnicos que presentan complejas relaciones entre ellos en las que esta identidad social ha cobrado una especial relevancia para el día a día de estas comunidades. Para alcanzar el objetivo propuesto utilizamos dos premisas de partida. Primero, defendemos que las identidades étnicas son ante todo ideacionales, relacionales y fluidas. Segundo, afirmamos que la cultura material participa de forma activa en los procesos de mantenimiento, negociación y subversión de estas identidades. En relación con estas ideas, tratamos de demostrar que la cultura material es parte consustancial de estos procesos $\mathrm{y}$, de forma consecuente, adopta estas mismas características.

Palabras clave: Identidad; etnicidad; tecnología; espacios domésticos; etnoarqueología.

\section{[en] "El techo de mi casa es particular": Ethnic Identities and Domestic Spaces in the Bunkpurugu-Yunyoo District (Northeast Ghana)}

\begin{abstract}
In this paper we investigate the profound and complex relationships that exist between ethnic identity and material culture, through an ethnoarchaeological study of the domestic spaces of several communities that inhabit the district of Bunkpurugu-Yunyoo (Northeast Ghana). This territory is inhabited by a number of ethnic groups that present a set of complex relationships. These relationships, and especially the day to day interactions between these groups are significantly influenced by social identity and will be discussed in detail. In order to explore these relationships, we used two initial premises. First, we defend the concept that ethnic identities are, above everything else, relational and fluid in nature. Second, we argue that that material culture plays an active role in the maintenance, negotiation, and subversion of those identities. In line with these viewpoints, we will demonstrate that material culture is in its essence part of these processes, and thus adopts the same characteristics.
\end{abstract}

Keywords: Identity; Ethnicity; Technology; Domestic Spaces; Ethnoarchaeology.

Sumario. 1. Introducción. 2. La importancia de las identidades étnicas en el Ne de Ghana en la actualidad. 3. Aproximación al concepto de identidad étnica y su relación con la cultura material. 4. Características generales de la vivienda en el Noreste de Ghana. 5. Vivienda, cultura material e identidades étnicas. 6. Conclusiones.

Cómo citar: Calvo Trias, M. et al. (2017): El techo de mi casa es particular: identidades étnicas y espacios domésticos en el distrito de Bunkpurugu-Yunyoo (noreste de Ghana). Complutum, 28(2): 399-416.

\footnotetext{
Este trabajo se inserta en el proyecto "Arqueología en la cuenca alta del Volta Blanco. N.E. Ghana”. Programa excavaciones arqueológicas en el exterior. Ministerio de Cultura 2010-2015.

2 ArqueoUIB Grupo de Investigación en Cultura Material y Gestión del Patrimonio Arqueológico. Universitat de les Illes Balears (España)

E-mail: manuel.calvo@uib.es; b) jaume.garcia@uib.es; c) davidjavaloyas@gmail.com; d) daniel_albero@hotmail.com
} 


\section{Introducción}

El presente artículo se basa en datos procedentes del trabajo desarrollado en el distrito de Bunkpurugu-Yunyoo; un territorio con una superficie aproximada de $1.000 \mathrm{~km}^{2}$ situado en el NE de Ghana y perteneciente, administrativamente, a la Northern Region (Fig. 1). Una de las principales características de este distrito es que se encuentra habitado por una amplia diversidad de comunidades etnolingüísticas entre las que, a nivel demográfico, destacan los grupos b'moba, komba ${ }^{3}$ y mamprusi ${ }^{4}$.

Los mapas etnolingüísticos de la zona muestran una distribución territorial de los diferentes grupos coherente y sin solapamientos (Fig. 2). Sin embargo, la realidad es más compleja ya que estos diferentes grupos cohabitan en el mismo territorio en una distribución tipo mosaico en la que no se observan límites espaciales claramente definidos. Si bien, los diversos grupos etnolingüísticos se articulan en torno a diferentes poblados con identidades étnicas relativamente homogéneas y mayoritarias (mamprusi, b'moba y konkomba).

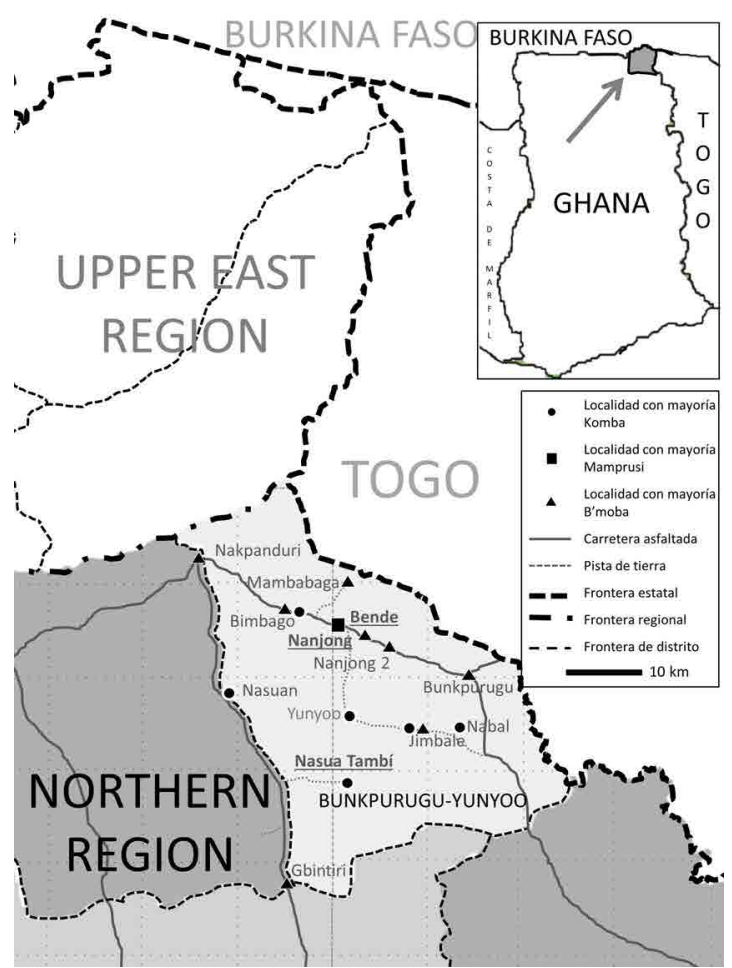

Figura 1. Mapa del área de estudio. En subrayado se marcan las localidades referidas en el texto.
Como es de suponer, las diferentes comunidades que habitan el territorio analizado presentan múltiples y complejas identidades sociales que se encuentran en permanente redefinición. Entre ellas podemos destacar las de género, estatus socioeconómico, familia, profesión, religión, etc. Sin embargo, en el presente estudio nos centraremos en cómo las casas de diferentes comunidades pertenecientes a los grupos étnicos komba, b'moba y mamprusi cobran un papel activo en la codificación, construcción, mantenimiento, negociación y subversión de las identidades étnicas.

Hemos decidido centrar nuestro trabajo en el estudio de los espacios domésticos al reconocer la importancia de las casas en las sociedades premodernas (p. ej. Bourdieu 1970; Drucker-Brown 2001; González-Ruibal 2006). Hay que destacar que en éstas no sólo se reflejan buena parte de los valores sociales que articulan a una determinada sociedad, sino que también juegan un papel activo en la propia configuración de la realidad social.

En el presente artículo reflexionamos sobre las profundas y complejas relaciones que

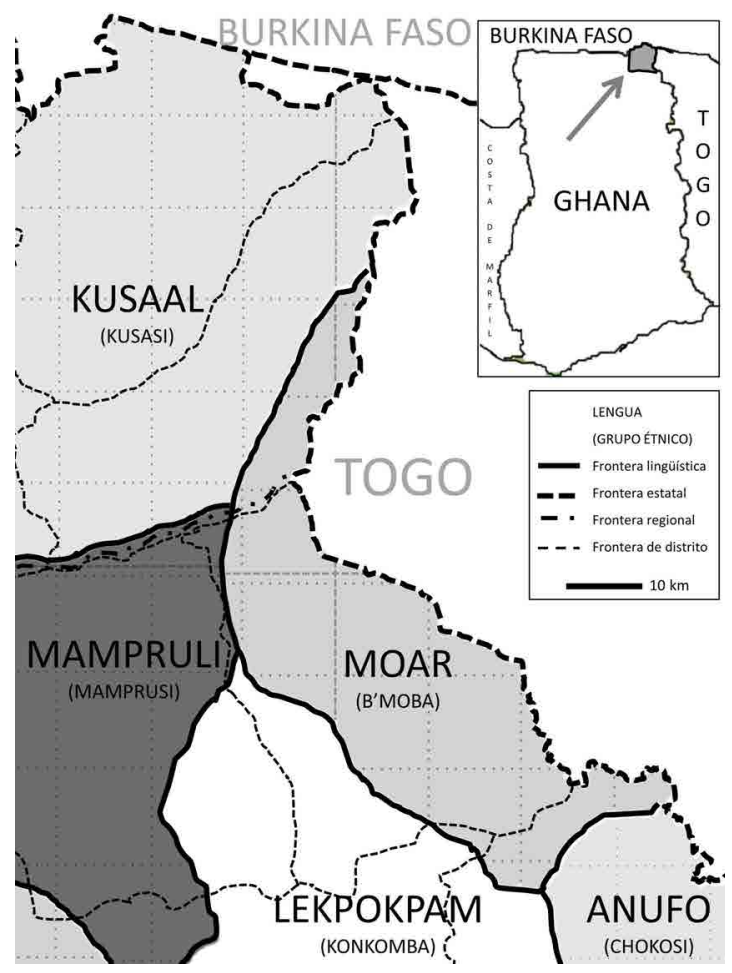

Figura 2. Mapa de la distribución de los grupos etnolingüísticos referidos en el texto (elaboración propia según Lewis 2009). 
se establecen entre las identidades étnicas y la cultura material. Para alcanzar este objetivo, partimos de dos premisas iniciales. En primer lugar, defendemos que las identidades étnicas son ante todo ideacionales, relacionales y fluidas. Segundo, creemos que la cultura material participa de forma activa en los procesos de mantenimiento, negociación y subversión de dichas identidades, por lo que es parte consustancial de estos procesos y, consecuentemente, incorpora parte de las dinámicas que se pueden observar en las identidades étnicas.

\section{La importancia de las identidades étnicas en el Ne de Ghana en la actualidad}

Aunque el eje central de nuestro trabajo se articulará en torno a la cuestión de las identidades étnicas, durante el mismo nos referiremos de forma general a diferentes identidades sociales. Desde nuestro punto de vista, la identidad étnica es una de las principales identidades sociales que articulan el día a día de las comunidades estudiadas. Por esta razón, defendemos que esta realidad influye profundamente en cómo estas comunidades hacen uso de la cultura material (Emberling 1997: 313).

El actual sistema político de Ghana es de tipo dual. Primero, se ha adoptado una república presidencialista democrática, estrechamente relacionada con los modelos occidentales europeos. Segundo, ésta se combina con un sistema tradicional de jefaturas, vinculado a los diferentes grupos étnicos que habitan el territorio ghanés. Este segundo sistema, aunque tiene sus orígenes en los momentos precoloniales, ha sufrido importantes cambios hasta la actualidad (Owusu-Mensah 2014). No es este el lugar para repasar la evolución histórica de ambos sistemas políticos y sus profundas y complejas relaciones en los procesos de etnogénesis (vid. García Rosselló et al. 2012; Calvo et al. 2014, 2016). Sin embargo, creemos necesario señalar algunos aspectos que demuestran la importancia en la actualidad de las identidades étnicas en el día a día de las comunidades estudiadas.

La presente Constitución ghanesa, aprobada en 1992, recuperó el Acta de Jefatura (Chieftaincy Act) de 1971 por la cual se aceptaba en el sistema político ghanés el papel de los jefes tradicionales como garantes de los valores y normas consuetudinarias. Así pues, éstos asumieron la jurisdicción sobre diversas prác- ticas tales como el matrimonio y el divorcio, las herencias y, especialmente, los derechos de propiedad de la tierra, recurso esencial entre las comunidades cuya base económica es esencialmente agrícola.

En el territorio estudiado, la aprobación de la Constitución de 1992 supuso la consolidación del poder mamprusi sobre el resto de grupos étnicos al ser el único de los principales grupos a nivel demográfico con representación plena en las estructuras políticas reconocidas por el Estado ghanés. Esta realidad, ha supuesto la existencia de fuertes tensiones en la zona (especialmente centradas en los derechos de propiedad de la tierra) que han provocado (y lo siguen haciendo) numerosos enfrentamientos, algunos de ellos muy violentos (p. ej. Pul 2003; Jönsson 2007).

A esta tensa situación política vinculada a los derechos de propiedad de la tierra se le ha añadido un nuevo elemento, como es el reciente proceso de comercialización de la tierra. La percepción entre muchos de los grupos étnicos del norte de Ghana de que la tierra es un derecho inalienable que pertenece "a los muertos, a los vivos y a los aún no nacidos" (Pul 2003: 62) está cambiando rápidamente como resultado del desarrollo de la economía de mercado. En esta nueva situación, la tierra se ha convertido en una importante fuente de ingresos tanto para los jefes tradicionales como para los grupos étnicos que detentan el poder político. En consecuencia, se ha reforzado la negación sistemática a que los grupos étnicos sin representación política, como los b'moba o los komba, puedan acceder al poder político tradicional. Esto ha agravado los conflictos ya que les impide participar en la toma de decisiones sobre la propiedad de la tierra.

\section{Aproximación al concepto de identidad étnica y su relación con la cultura material}

Se han utilizado infinidad de aproximaciones para precisar cuál es la esencia de un grupo étnico y especificar aquellos elementos comunes que comparten los individuos que lo conforman. Entre los más citados se encuentran aspectos como el lenguaje, la cultura, la religión, la cultura material, la existencia de un etónimo, un determinado fenotipo o de una ascendencia común (biológica o percibida), la adscripción a un territorio determinado, la auto-concepción como grupo, etc. (Smith 
1986: 22-30; Renfrew 1987: 2; Hall 1997:21; Knapp 2001: 31).

A pesar de que algunos autores (p. ej. Meskell 2001: 189) defienden que nunca ha habido consenso sobre lo que significa etnicidad y que ésta se ha utilizado únicamente para contraponer individuo y grupo, nuestro trabajo se basa esencialmente en la propuesta de Weber (1968 [1922]: 289), ampliamente aceptada en la actualidad (Eriksen 1993: 12; Emberling 1997: 302-303; Jones 1997: xii; Meskell 2001: 190; Sweeney 2009; Curta 2014: 2508; cf. Smith 1986: 22-30; Hall 1997: 25). Esta propuesta defiende que la etnicidad es un tipo de identidad colectiva caracterizada por un parentesco metafórico o ficticio. Ésta se sustenta en las similitudes bien de tipo material y/o de costumbres y/o en la existencia de memorias de colonización y migración. No obstante, la realidad genealógica y biológica de tales reivindicaciones es irrelevante, lo que importa es que la pretensión de un ancestro común es consensuada.

Sin embargo, más allá de la propia definición de grupo étnico utilizada, para el estudio propuesto nos resulta más operativo analizar cuáles son las características que presentan las identidades sociales (incluida la étnica) y como éstas se construyen y activan.

Uno de los modelos más aceptados, y que nosotros incorporamos en nuestro discurso, se conoce con el nombre de identidad situacional (Shennan 1989: 16; Knapp 2001: 32). Esta corriente de pensamiento trata de superar dicotómicas posiciones anteriores (primordialistas e instrumentalistas) en base a tres características esenciales de lo que se caracteriza como identidad: la autoconsciencia, la relacionabilidad y la fluidez.

El primer componente podríamos denominarlo como la concepción autoconsciente $e$ ideacional de la identidad. Se propone que las identidades son "constructos sociales subjetivamente percibidos" (Hall 1997: 19). De esta manera, se define el carácter de estos grupos como una categoría emic de adscripción sin que haya una necesaria relación directa y univoca con las discontinuidades culturales percibidas por los observadores. En definitiva, lo que caracteriza a un grupo identitario sería la capacidad de los individuos que pertenecen a él de definirse como tal, de forma consciente. Es decir, lo que es relevante es la capacidad de identificarse como distintos frente a otros grupos, minimizando, por tanto, las diferencias existentes dentro del propio grupo.
Esto puede parecer problemático si nuestra estrategia se basa en el estudio de la cultura material. Esto se debe a que si bien las diferencias existentes en la cultura material y, por tanto, en las prácticas sociales, son parte de los rasgos que se asocian a grupos identitarios, esto no ocurre en todos los casos ni es, nunca, suficiente. Esta perspectiva continúa aceptando que los individuos marcan su sentido de pertenencia a un grupo a través de las prácticas culturales compartidas. Sin embargo, las prácticas no son identidades, y mientras que las personas pueden adoptar prácticas afiliadas a un grupo, esto no es señal de su adhesión automática a ese grupo (Casella y Fowler 2004: 7).

La segunda característica esencial de las identidades sociales es que son siempre relacionales o interaccionales. Este carácter interaccional defiende que los componentes físicos e ideológicos de un grupo identitario deben concebirse en términos de sus límites, es decir, con respecto a las distinciones entre grupos. Como expone Barth (1969: 15): "son los límites los que definen a un grupo, no los rasgos culturales que encierra". En cierta manera, las identidades serían un proceso efímero, en continua construcción, cuyo principal elemento sería la negociación de la diferencia (Sweeney 2009: 104). Por tanto, las diferentes identidades sociales (étnicas, de género, edad, rango, etc.) están en estrecha conexión tanto entre ellas (Meskell 2002) como con las que presentan otros grupos a los que se oponen y diferencian.

Finalmente, la tercera característica es el aspecto fluido de las identidades sociales. Estas interpretaciones enfatizan la plasticidad de las identidades y otorgan agencia a los actores sociales que pueden resaltar y/o modificar sus múltiples identidades en función del contexto y sus intereses. Debido a que los actores sociales poseen numerosas identidades, en cualquier momento de su vida éstas se pueden ir activando en función del contexto en el que se encuentren. Por esto, Casella y Fowler (2004: 2) hablan de las identidades como experiencias transitorias a nivel sincrónico y diacrónico, en el sentido que son múltiples en un momento determinado y van cambiando a lo largo del tiempo.

Junto a todo ello, debemos reflexionar sobre cómo se van configurando las identidades sociales. Para ello, partiremos de las premisas teóricas desarrolladas por la "Teoría de la Práctica” de Bourdieu (1977, 1990). 
De entrada, este modelo señala que la práctica individual es el lugar en el que se producen los procesos estructurados que crean y recrean la sociedad (Yaeger y Canuto 2000: 3). En este sentido, actuarían tanto los fenómenos de habitus (Bourdieu 1977, 1990) como los de agencia (Hall 1997: 18). Así pues, el cambio social se interpreta en términos de las continuas transformaciones en las disposiciones estructuradas del habitus a través de procesos de agencia que ocurren en los cambiantes contextos de la práctica social.

En segundo lugar, esta aproximación tiene diversas ventajas a la hora de entender y analizar la complejidad de la conformación, mantenimiento y cambio de las identidades colectivas, entre ellas la identidad étnica. De esta manera, permite entender "cómo la gente llega a reconocer los rasgos que tiene en común" (Bentley 1987: 27). La "Teoría de la Práctica" señala que la identidad no surge como un reflejo pasivo de las semejanzas y diferencias existentes en las prácticas culturales - como defendían las aproximaciones primordialistas. Por otra parte, critica que las identidades se puedan concebir como farsas construidas y mantenidas conscientemente para conseguir ciertos intereses políticos y económicos, como argumentaban las corrientes instrumentalistas (Bentley 1987: 26; Jones 1997: 92; Hall 1997: 17).

Por el contrario, se argumenta que las identidades colectivas surgen de "las disposiciones subliminales y compartidas del habitus que modelan y son modeladas por los elementos objetivos comunes de la práctica" (Jones 1997: 90). Esto supone reconocer que los recursos simbólicos identitarios no son arbitrarios, sino que están íntimamente ligados con el mundo experimentado y práctico que contiene significados relevantes que contribuyen a modelar la interacción y, al mismo tiempo, limitan el número de opciones en la producción de símbolos identitarios (Eriksen 1992: 45). Sin embargo, y teniendo en cuenta la concepción relacional que defendemos para la identidad, de esta premisa no se deriva que la existencia de un habitus compartido implique la existencia de una identidad similar. Por el contrario, siguiendo a Jones (1997: 94) defendemos que la identidad es ante todo una "conciencia de la diferencia". Esta surge, en momentos en los que comunidades que presentan habitus y prácticas sociales muy distintas entran en contacto. En estas situaciones, ciertos aspectos inconscientes de la propia sociedad que apa- recían como naturalizados se trasladan a un nivel discursivo y explícito, lo que favorece la aparición de una concepción auto-reflexiva de las diferencias existentes en los habitus de ambas comunidades.

En definitiva, la confluencia de habitus, agencia y práctica permite reconocer la flexibilidad y fluidez tanto de las identidades como de los símbolos asociados a ellas. Es decir, permite entender las identidades no como entidades estáticas, sino como procesos siempre activos, que se generan, negocian, mantienen y modifican a través de las prácticas diarias.

Por otra parte, la implementación de la "Teoría de la Práctica" tiene, a su vez, sustanciales implicaciones en lo que se refiere a la concepción de la cultura material y su papel en la configuración y negociación de las identidades. Por tanto, como ya proponía el seminal trabajo de Jones (1997), permite interesantes enfoques en el estudio de ésta.

En primer lugar, utilizar los conceptos de habitus y agencia supone un importante cambio con respecto a los modelos anteriores ya que permite entender por qué la distribución de rasgos de cultura material no tiene por qué corresponder a límites identitarios. (Jones 1997: 92). En segundo lugar, supone un importante énfasis en la cultura material como agente activo en la configuración de las sociedades lo que, como ya hemos dicho, es parte esencial de nuestro corpus teórico. Según esta corriente teórica, la cultura material presenta un funcionamiento muy distinto y complementario al que presentan los aspectos discursivos. Este funcionamiento, se funda en la práctica y permite enlazar el uso de la cultura material con la concepción ideacional, fluida, relacional y permanentemente negociada de la identidad situacional. Así pues, la cultura material pasa a ser una parte esencial del proceso de generación, negociación y mantenimiento de las identidades. Es a través de su utilización reiterativa en la práctica cotidiana que los agentes sociales pueden activamente añadir o modificar sus identidades sin negar las identidades pasadas (Smith 2007: 416-7).

Dentro de este esquema teórico utilizaremos los conceptos de marcador étnico pasivo y marcador étnico activo. Estas dos herramientas conceptuales nos permitirán entender mejor el funcionamiento de la cultura material en los procesos identitarios en general y en los étnicos en concreto. Entendemos como marcador étnico, en una organización social 


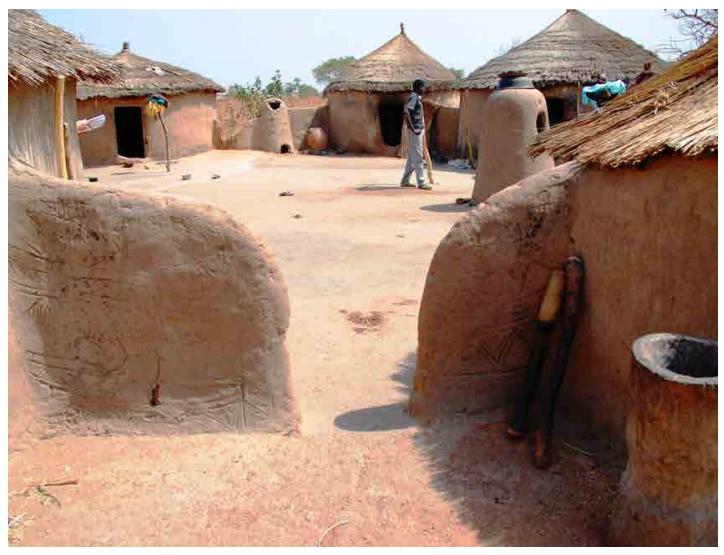

Figura 3. Entrada, patio central, muro perimetral y algunas habitaciones (Casa Jijirs, etnia komba, en Nasua Tambí).

determinada, cualquier elemento material que permite a los actores sociales identificar una manifestación concreta de la identidad étnica (Reher 2011: 661), en el sentido que supone un reconocimiento de la diferencia entre distintos grupos étnicos. A su vez, diferenciamos entre marcadores étnicos pasivos y activos. Esta distinción requiere, a nuestro modo de ver, de ciertas clarificaciones para evitar confusiones.

En primer lugar, la distinción entre marcadores activos y pasivos no la planteamos desde el plano ontológico, sino que nos referimos al campo de la acción simbólica. Así pues, estamos de acuerdo en que la cultura material es siempre activa en los procesos sociales, independientemente de los procesos de acción simbólica (p.ej. Olsen 2003, 2010; Latour 2008; Hodder 2012; González-Ruibal 2014). En segundo lugar, al referirnos al campo simbólico no estamos negando la máxima postprocesual que afirma que la cultura material no es únicamente reflejo pasivo de la sociedad, sino que siempre tiene un papel activo en la conformación de esta (Hodder 1982).

De esta manera, la distinción entre marcadores étnicos activos y pasivos hace referencia a si éstos son manipulados conscientemente (activos) o no (pasivos) por los actores sociales en la negociación de las identidades (étnicas). Desde nuestro punto de vista, esta distinción es relevante ya que mientras que los pasivos nos remiten a un habitus determinado, los activos nos muestran la agencia de los actores sociales.

\section{Características generales de la vivienda en el Noreste de Ghana}

Como hemos señalado previamente, para abordar el tema de la construcción de las identidades étnicas a partir de la cultura material desde el marco teórico expuesto, hemos realizado un estudio de la organización y uso del espacio doméstico de los grupos étnicos komba, b'moba y mamprusi.

De este modo, hemos analizado cómo se construyen las viviendas, la localización de los diferentes ámbitos y su funcionalidad. También hemos estudiado la existencia de distinciones entre espacios públicos y privados, entre ámbitos femeninos y masculinos, y entre grupos de edad. Asimismo, hemos examinado la ubicación y uso de los elementos materiales relacionados con las tareas de mantenimiento del grupo, almacenaje o el culto, tales como pequeños altares, mezquitas, enterramientos, etc. El análisis de todos estos aspectos también se ha relacionado con la esfera social en lo que se refiere a la cosmovisión, los distintos tipos de identidades (género, étnica, religiosa, etc.), organización social, tareas productivas desarrolladas en el ámbito doméstico, etc.

Para la documentación de todos estos aspectos hemos implementado una doble estrategia:

1.- Entrevistas generales sobre la vivienda, la familia y la esfera social más amplia. En estas entrevistas se ha seguido un formato de conversación abierta con el objetivo de obtener información relativa a la familia que habita en ella. En estas entrevistas, se han planteado aspectos que, si bien no tienen que ver directamente con la cultura material de la casa, se configuran como elementos clave para entender la organización socioeconómica y cultural de cada una de las familias estudiadas.

2.- Información relativa a la vivienda a través de a) fichas de análisis para cada una de las estancias y áreas de la casa, b) croquis donde se plasma la localización de las diferentes unidades que configuran el espacio y c) documentación fotográfica de cada una de las estancias.

El análisis objeto de este estudio se ha centrado en las poblaciones de Nasua Tambí (de mayoría komba), Nanjong (de mayoría b'moba) y Bende (de mayoría mamprusi) (Fig. 1). Nasuá Tambí se sitúa a unos escasos $24 \mathrm{~km}$ de las otras dos localidades, que se encuentran separadas entre sí por unos cientos de metros; todas las localidades se localizan en el extre- 
mo NE de la Northern Region, en el distrito de Bunkpurugu-Yunyoo. Durante las diferentes campañas realizadas se han estudiado 20 casas: diez mamprusi en Bende, cinco b'moba en Nanjong y cinco komba en Nasuá Tambí.

Los espacios domésticos que encontramos en las comunidades del noreste de Ghana son relativamente homogéneos (Drucker-Brown 2001: 669). Se trata de recintos habitacionales (compounds), construidos en adobe y/o tapial y vegetales trenzados para techar. Están organizados alrededor de un patio central comunitario de tendencia circular, en el cual se desarrolla buena parte de la vida diaria. La naturaleza concreta de estos espacios está estrechamente relacionada con la organización social básica de estas comunidades. Así pues, los distintos grupos étnicos se organizan en familias extensas que siguen un modelo patriarcal, virilocal y poligínico. De esta manera, a menudo, conviven en una misma vivienda todos los hijos y nietos del landlord, el jefe de la familia que hereda la propiedad de la tierra y la vivienda por vía masculina, con sus respectivas mujeres e hijos.

De forma general, las casas están formadas por habitaciones circulares de unos $3 \mathrm{~m}$ de diámetro cuyas puertas se abren al patio central (Fig. 3). Un muro con una altura que oscila de 1 a 1,5 m une las habitaciones por su parte exterior y cierra el patio central dejando una única entrada, normalmente orientada al oeste (Fig. 3). Adosadas a las habitaciones destaca la presencia de diferentes baños (Fig. 4) y al frente de las habitaciones femeninas las cocinas (Fig. 5a). Otro grupo de estructuras que encontramos asociados a las casas, normalmente situados en el exterior, son aquellos dedicados a labores productivas como gallineros y establos para animales de pequeño tamaño, graneros, leñeros o ponedores de huevos para las gallinas (Fig. 6).

Otro aspecto esencial de estos espacios domésticos es la existencia de zonas diferenciadas para los distintos sub-grupos que articulan la familia extensa. Por un lado, tenemos a los diferentes adultos masculinos, representados por el landlord y sus hijos mayores ya casados. Estos ocupan dormitorios individuales y, aunque no siempre, suelen tener baños también individuales.

Por su parte, las mujeres casadas que viven dentro del recinto - las esposas del landlord y de sus hijos mayores - también tienen dormitorios individuales que, sin embargo, compar-

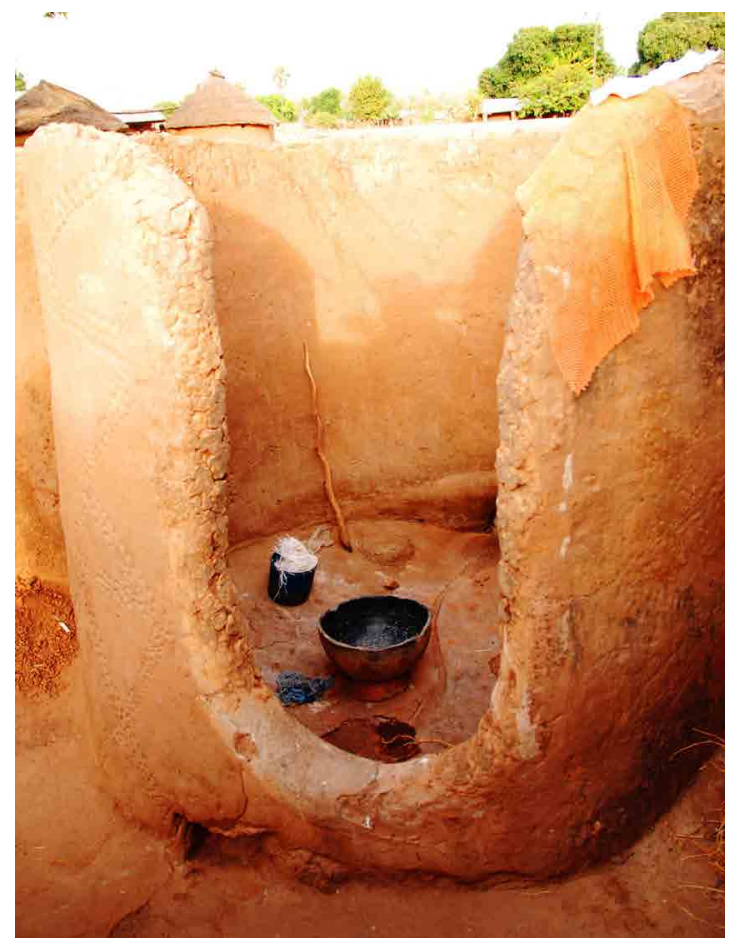

Figura 4. Baño (Casa Banjua, etnia mamprusi, en Bende).

ten con sus hijos e hijas en edad infantil. Los dormitorios, tanto los de los hombres como los de las mujeres, son espacios privados a los que normalmente no acceden el resto de componentes de la familia. Las mujeres, además, poseen generalmente dos cocinas, una exterior cerca de su dormitorio (Fig. 5a) y una interior dentro del mismo (utilizada preferentemente en la estación lluviosa) (Fig. 5b). Las cocinas son de uso individual y en ellas preparan los alimentos para su prole y, en ocasiones para su marido (éste puede comer de cualquiera de los hogares de sus diversas mujeres). Como regla general, las mujeres casadas también tienen un baño propio que comparten con su descendencia (Fig. 4).

En contraposición a estos espacios individuales, en el recinto habitacional también hay diversos espacios de carácter comunal. En primer lugar, tenemos el patio central en el que, como hemos señalado anteriormente, se realiza buena parte de la vida diaria: preparación de alimentos, consumo de comida, charlas informales, actividades artesanales, etc. También en el exterior y cerca de la entrada del compound, hallamos un pequeño porche, realizado con madera de caoba, que constituye el punto de 

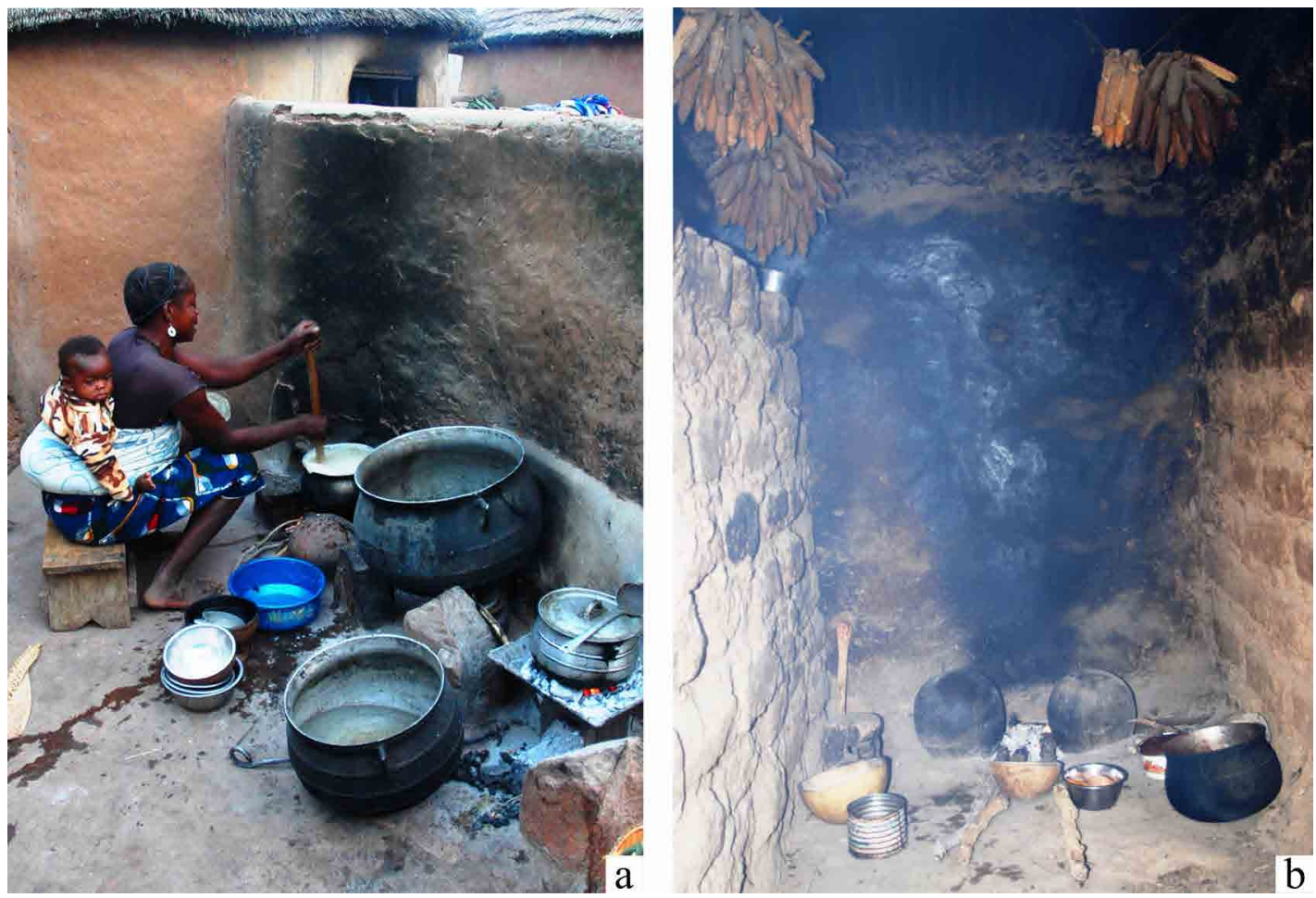

Figura 5. Cocina exterior (Casa Pusuara, etnia mamprusi, en Bende). b- Concina interior (Casa Buesa, etnia komba, en Nasua Tambí).
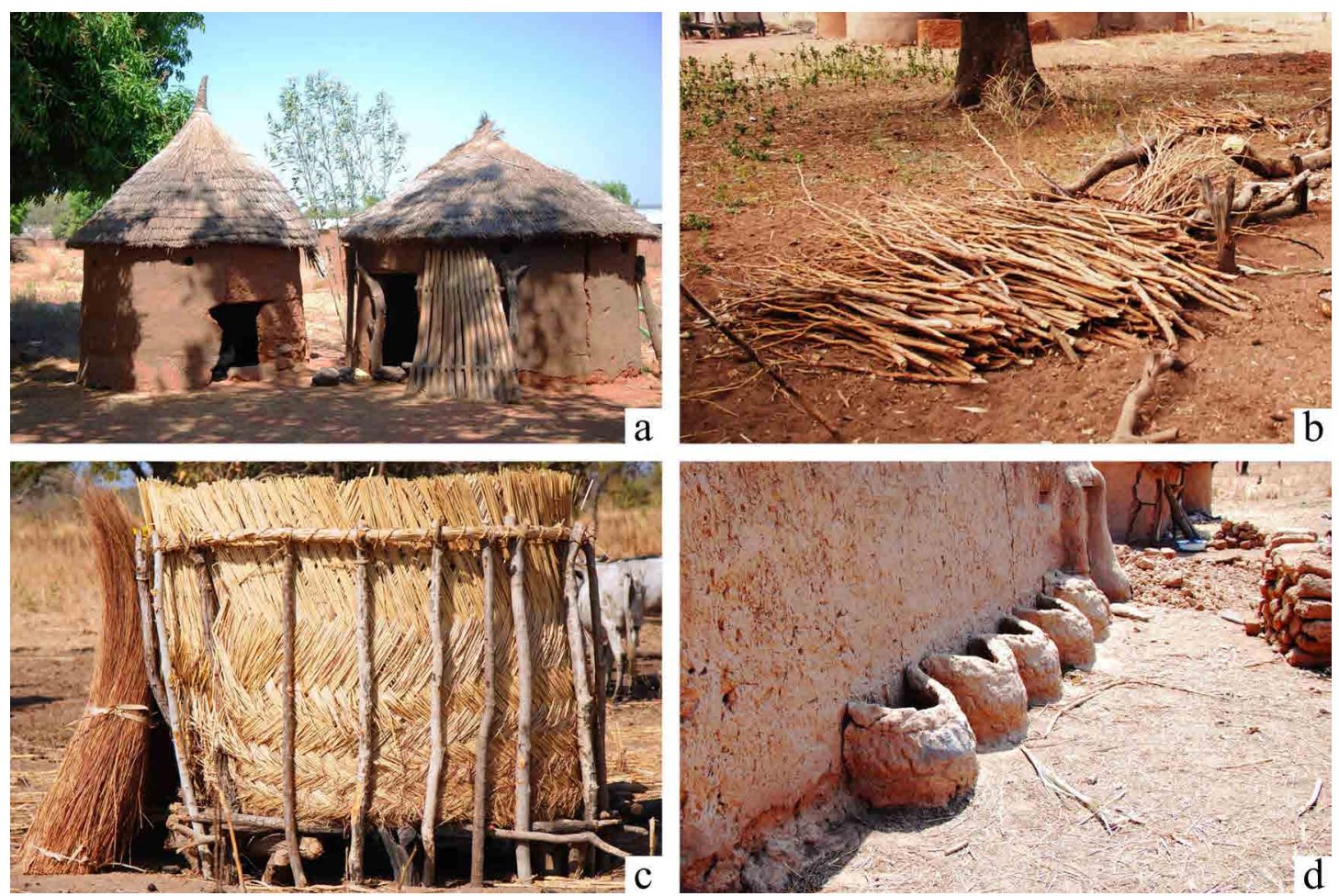

Figura 6. a- Gallinero (Casa Dama, etnia mamprusi, en Bende); b- Leñero (Casa Demonsu, etnia mamprusi, en Bende); c- Granero (Casa Buesa, etnia komba, en Nasua Tambí); d- Ponedero para gallinas (Casa Buesa, etnia komba, en Nasua Tambí). 


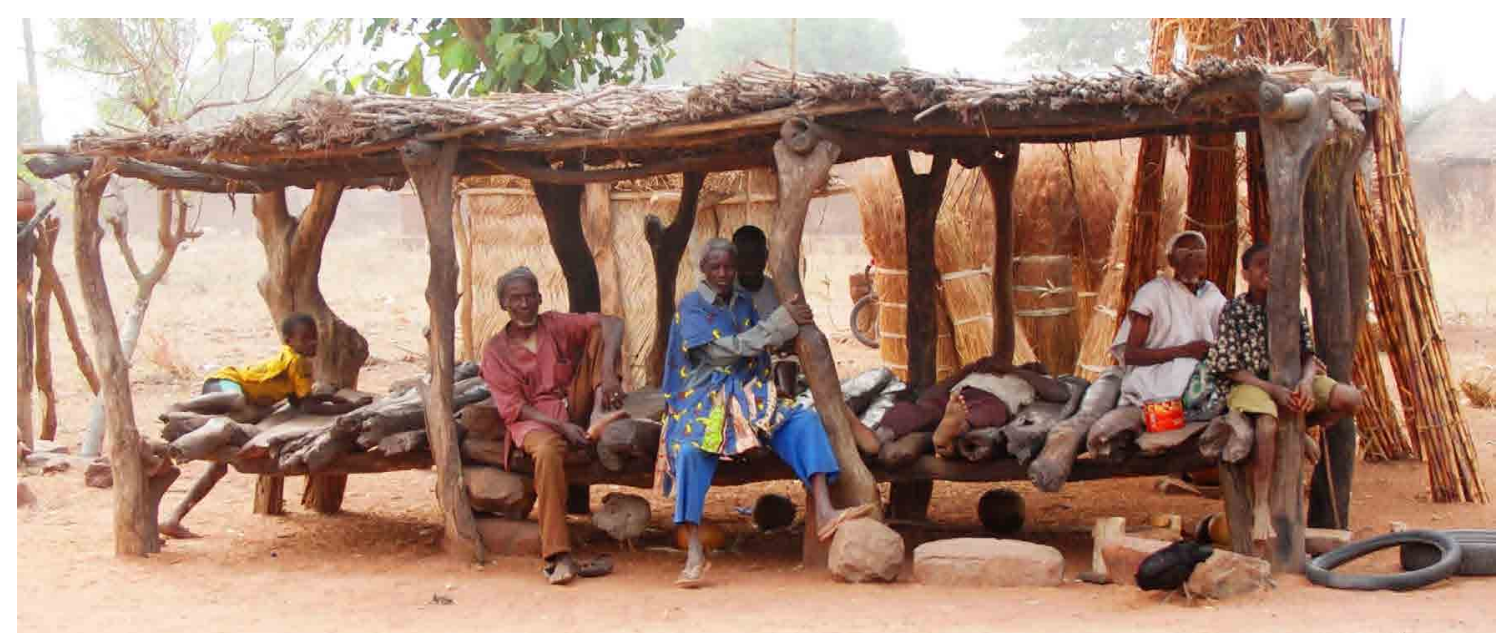

Figura 7. Porche exterior de caoba (Casa Demonsu, etnia mamprusi, en Bende).

reunión preferido por los hombres de la casa (aunque los niños, niñas y las mujeres también lo usan). Bajo este porche se reúnen con los diferentes visitantes que pasan por los exteriores de la casa (Fig. 7).

\section{Vivienda, cultura material e identidades étnicas}

Una de las preguntas que realizábamos a nuestros informantes con la intención de entender la relación existente entre la cultura material de las casas y las diferentes identidades étnicas de las comunidades que habitan en la zona era si eran capaces de distinguir el grupo étnico al que pertenecían las diferentes viviendas. Tanto en el caso de los mamprusi como en el de los komba y los b'moba la respuesta fue afirmativa: el techo de las habitaciones que conforman los compounds les permitía distinguir las viviendas de cada grupo étnico. Los informantes señalaban que los mamprusi utilizaban una cuerda para realizar el remate del vértice cónico del techo; que los b'moba utilizaban las cañas procedentes del mijo como primera capa del techo y que esta formaba un alero claramente visible; y que los komba utilizaban un poste central para dar fuerza al conjunto y que éste sobresalía por encima del techo (Fig. 8). Analizado el conjunto de unidades habitacionales de los tres poblados en los que centramos nuestro trabajo, pudimos concluir que si bien este era el patrón que funcionaba en la mayoría de ocasiones, es necesario recalcar que existían algunos casos en los que esta regla general no se cumplía.

Atendiendo a los datos reunidos sobre esta cuestión nos planteamos la posibilidad de que estuviésemos ante un marcador étnico. En
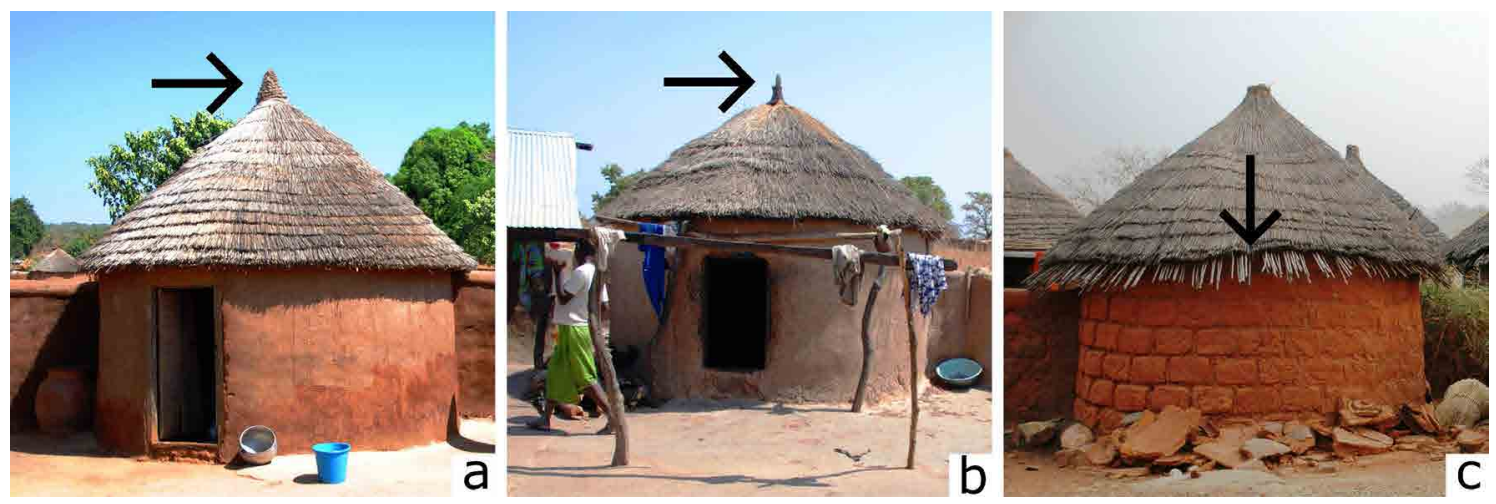

Figura 8. Techos tradicionales de las habitaciones. a- Mamprusi (Casa Dama, en Bende); b- Komba (Casa Jijirs, en Nasua Tambí); c- B'moba (Casa indeterminada, Nanjong I). 
cualquier caso, se trataría de un marcador étnico de tipo pasivo puesto que al preguntar a los diferentes informantes la razón por la cual utilizaban las diferentes soluciones técnicas específicas en la fabricación de estos elementos la respuesta era invariablemente: "porque es así cómo se hace". En ningún momento señalaron que se tratara de una acción consciente mediante la cual expresaban su identidad étnica, sino que respondía a una manera concreta de construir estos elementos que se había trasmitido de generación en generación a través de redes específicas de enseñanza-aprendizaje.

Entre estos grupos, la construcción de la casa es una tarea comunal en la que participan no sólo los futuros residentes, sino en la que también ayudan otros miembros de la comunidad, especialmente aquellos vinculados por lazos familiares y/o de amistad. A lo largo del proceso de construcción se observa una clara separación del trabajo en función del género. La construcción de la estructura básica de la vivienda, el trabajo con los adobes y el tapial, y el techado de las habitaciones son tareas eminentemente masculinas. En cambio, las mujeres y niñas participan únicamente en el revestimiento y decoración de las paredes, los suelos y las habitaciones.

Así pues, teniendo en cuenta que estamos ante sociedades patriarcales y virilocales, en las que la identidad étnica de los hombres se transmite por vía paterna y que éstos sitúan su residencia cerca de la vivienda del padre, el conocimiento tecnológico de la construcción de las viviendas se transmite, al igual que la filiación étnica, por vía patrilineal. El resultado de esta realidad es la generación de un habitus tecnológico de adscripción étnica, lo que explicaría las diferencias materiales existentes entre los techos de los diferentes grupos étnicos asentados en la zona.

En este sentido, y atendiendo a lo expresado por los informantes, los techos podrían ser considerados como marcadores étnicos de tipo pasivo, ya que no existe una voluntad consciente de expresar la pertenencia a un grupo étnico a través de la construcción y uso de estos elementos. Sin embargo, a pesar de la ausencia de una manipulación consciente, parece claro que los actores sociales son capaces de distinguir la etnia de sus constructores a través de este elemento material. Esto es, de los habitantes masculinos de la casa que son los que proporcionan la filiación étnica a la familia que la habita. En definitiva, los techos son elementos que participan en la construcción de la identidad étnica en tanto en cuanto configuran la percepción de la diferencia entre los distintos grupos.

Hasta este momento nuestro caso de estudio puede parecer algo simplista, mecanicista y en plena contradicción con nuestra propuesta teórica. Efectivamente, la realidad es más compleja, diversa y dinámica. Así pues, desde la concepción de que las identidades étnicas son autoconscientes e ideacionales, relacionales y fluidas y de que la cultura material desempeña un papel clave en su configuración defendemos que la relación identificada entre los techos de las casas y los tres principales grupos étnicos analizados no puede cosificarse ni plantearse de forma estática y mecánica. A continuación, trataremos de abordar estas características mediante el uso de cuatro análisis específicos que permitirán añadir mayor profundidad a esta primera reflexión.

En primer lugar, debemos tener en cuenta que estos grupos étnicos ocupan amplios territorios y engloban a un gran número de personas. En consecuencia, no podemos dar por sentado que todos sus miembros compartan las mismas manifestaciones e ideas en relación con su identidad étnica. En este sentido, creemos necesario remarcar que nuestro caso de estudio no hace referencia de forma global a todos los miembros de estos grupos étnicos sino única y exclusivamente a aquellos que habitan de forma conjunta en el distrito de Bunkpurugu-Yunyoo. El elemento clave en la configuración de las identidades étnicas de estas comunidades es precisamente la cohabitación en un mismo territorio en el que existen importantes conflictos vinculados al control del poder político y la propiedad de la tierra, lo que supone la activación de las diferentes identidades étnicas. La conceptualización de éstas en otras zonas es sustancialmente diferente y, por tanto, también lo acaban siendo las relaciones que se establecen con la cultura material.

En segundo lugar, es importante remarcar que la relación entre techos e identidades étnicas se refiere de forma específica a los individuos de género masculino. La relación entre cultura material e identidad étnica asociada a las mujeres que habitan las diferentes casas es sustancialmente diferente. En primer lugar, estamos ante comunidades poligínicas, por lo que un mismo hombre puede tomar diferentes esposas que pueden ser de diferente etnia. Segundo, son sociedades virilocales, en las que 


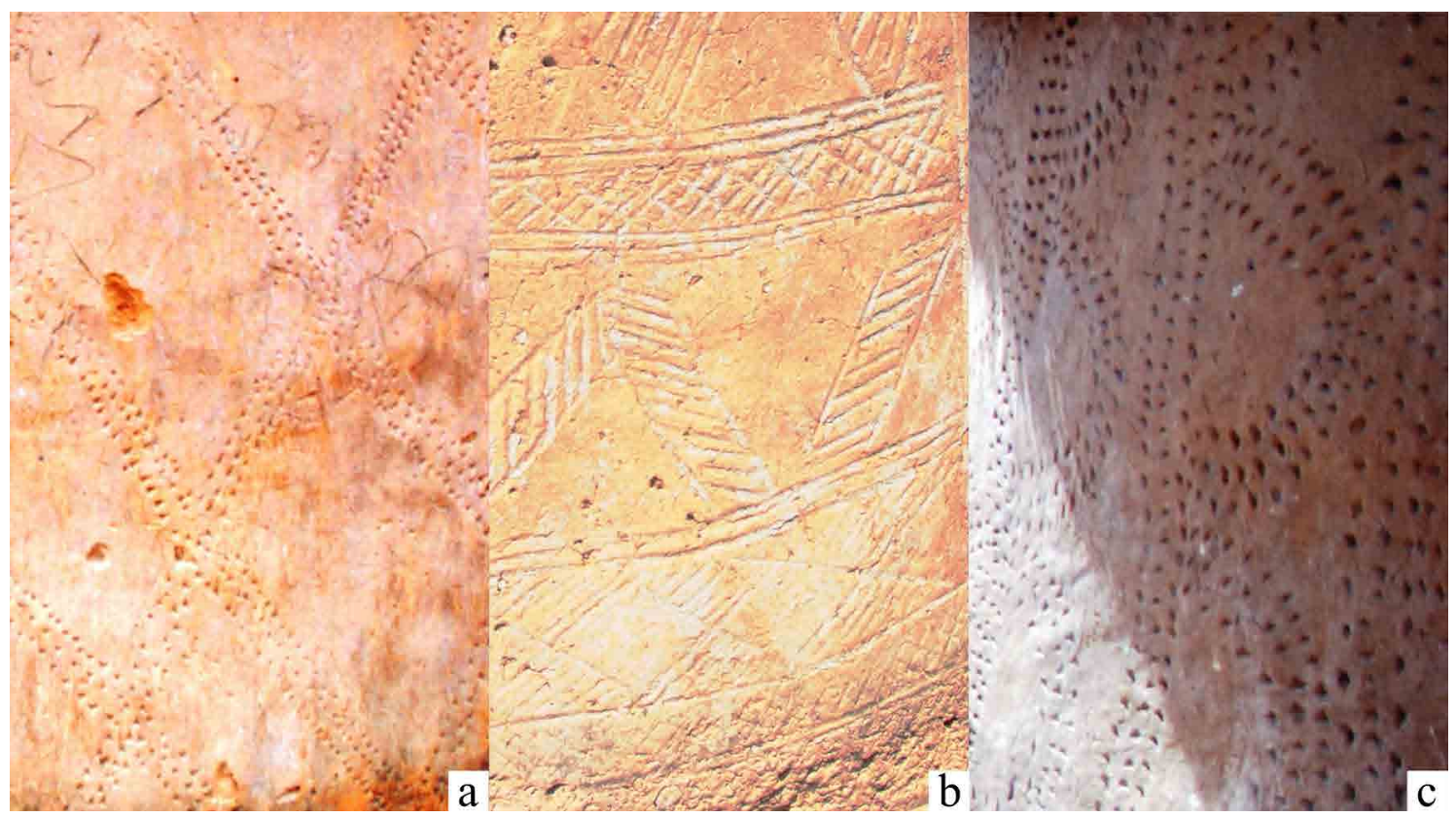

Figura 9. Motivos decorativos en las paredes de las habitaciones. a- Mamprusi; b- Komba; c-B'moba.

las mujeres al casarse abandonan la casa del padre para irse a vivir a la de su marido (o a la del padre del marido). De esta manera, como señalaba González-Ruibal de forma general para las sociedades africanas (2003: 131-132), en la mayoría de las ocasiones las mujeres se encuentran "con un hogar ya construido y estructurado sobre el cual su capacidad de expresión material es mínima" así, "las mujeres se ven desposeídas del derecho a gestionar el espacio (construir, reconstruir, destruir, organizar)".

Sin embargo, a pesar de estas limitaciones, las mujeres de estas comunidades tienen la capacidad de ejercer cierto poder en la definición material del espacio habitado. En otro trabajo (Calvo et al. 2016) demostramos cómo la identidad étnica de las mujeres (que viene marcada por el padre de éstas) influye de forma sustancial en el tipo de cerámicas consumidas $\mathrm{y}$, por tanto, presentes en el espacio doméstico. De esta manera, las mujeres marcan sus espacios con los diferentes tipos de cerámica consumida, diferenciándose claramente de mujeres de otras etnias o de la propia filiación étnica de la casa que se relaciona con la etnia del landlord.

En esta misma dirección, en las diferentes comunidades analizadas, las mujeres participan únicamente en el revestimiento de las paredes y suelos de la casa. Una vez los hombres han acabado con la construcción de las paredes de la casa y han techado las habitaciones, las mujeres homogeneizan toda la superficie construida aplicando una mezcla de tierra diluida con agua. En este momento son libres de decorar sus propias habitaciones. En este proceso, utilizan patrones decorativos propios de su grupo étnico, que no tiene por qué coincidir con el grupo étnico del marido ni, en consecuencia, con el de la unidad familiar (Fig. 9).

Estos tipos decorativos también pueden ser considerados como marcadores étnicos pasivos. Aunque hemos documentado que las diferentes mujeres pueden distinguir claramente entre los diferentes motivos decorativos, cuando les preguntamos por qué utilizan éstos y no otros señalan que son los que les gustan y los que han aprendido a hacer. Es decir, encontramos otra vez una plasmación concreta y distinta de un habitus tecnológico dependiente de las redes de aprendizaje y de la vinculación étnica familiar que se convierte en marcador étnico pasivo, al ser fácilmente reconocible por los actores sociales. Así pues, un ejemplo interesante tiene lugar cuando una mujer komba contrae matrimonio con un hombre mamprusi. En estos casos, aunque la casa la construyen hombres mamprusi, siguiendo patrones mamprusi, las mujeres komba la decoran utilizando patrones decorativos propios de su identidad 
étnica komba, en una clara acción de apropiación (pasiva) del espacio construido.

Es interesante realizar una reflexión conjunta sobre la visibilidad de los dos marcadores étnicos pasivos identificados en los espacios domésticos: los techos, importantes para la esfera masculina y la filiación étnica del grupo familiar y las decoraciones de las habitaciones, relevantes para las identidades étnicas de las mujeres. Por una parte, tenemos que los techos de las habitaciones son uno de los elementos más conspicuos de toda la vivienda, pudiendo ser identificados desde el exterior a cierta distancia. En contraposición, las decoraciones realizadas por las mujeres en las paredes de las habitaciones se sitúan, en la mayoría de los casos, en los vanos de las puertas o en las paredes que miran hacia el patio de la vivienda, en el interior, por lo que su visibilidad se reduce al interior de la vivienda.

A nuestro modo de ver, esta disposición visual diferencial se puede relacionar con las esferas sociales prominentes a cada género. Pues, en las comunidades estudiadas los hombres ocupan de forma hegemónica la esfera de la vida pública, mientras que el papel de las mujeres es clave en el espacio doméstico.

Un tercer ejemplo de la compleja relación existente entre la cultura material y la concepción autoconsciente e ideacional, relacional y fluida de las identidades sociales se vincula a una serie de innovaciones tecnológicas que se introdujeron en la construcción de las habitaciones de las casas. Hace unos 45 años en la zona estudiada (Drucker-Brown 2001: 671) se comenzaron a utilizar planchas metálicas (zinc corrugado) que sustituyen, en algunos casos, a las cubiertas vegetales tradicionales en el techado de las estructuras. Además, también se observa la introducción de ladrillos industriales de hormigón que reemplazan los materiales tradicionales (adobe/tapial) o de pinturas industriales que sustituyen otros pigmentos naturales. La introducción de estos nuevos materiales ha supuesto (y sigue haciéndolo) diferentes cambios en el ámbito de los espacios domésticos, tanto a nivel social como material, influyendo decisivamente en la materialización de las identidades étnicas a través de las viviendas.

De entrada, la introducción de esta nueva tecnología y materiales en el techado de las habitaciones ha implicado la adopción de plantas rectangulares con tejados a dos aguas. $\mathrm{Al}$ preguntar a los informadores por este hecho siempre responden que se debe a que es imposible construir habitaciones de planta circular utilizando estos materiales ${ }^{5}$. La adopción de esta nueva tecnología no modifica de manera sustancial la organización espacial de los complejos habitacionales (Drucker-Brown 2001), que conservan la estructuración general y sobre todo la importancia del patio central. Sin embargo, sí definen nuevos patrones de visibilidad entre el interior y el exterior de la casa. Así, en los complejos domésticos con una presencia importante de nuevos materiales, y de habitaciones de tipo cuadrangular, se reduce de forma notable la visibilidad entre el exterior $\mathrm{y}$ el interior.

Otro aspecto importante a considerar se relaciona con la organización social del trabajo y las redes de transmisión del conocimiento tecnológico. Como señalamos anteriormente, la construcción tradicional de las casas (incluyendo el techado de las habitaciones) es una tarea comunal desarrollada por los hombres que habitarán la casa con ayuda de otros hombres de la comunidad unidos por lazos familiares y/o de amistad. Éstos comparten un mismo habitus tecnológico trasmitido de generación en generación a través de redes específicas de aprendizaje patrilineal. En definitiva, el habitus tecnológico del techado de habitaciones está estrechamente ligado con la identidad étnica.

Sin embargo, la adopción de estos nuevos materiales y técnicas constructivas supone una ruptura importante en la tradición tecnológica. Puesto que esta nueva situación implica la contratación de mano de obra especializada cuyo habitus tecnológico se desmarca de las redes tradicionales de aprendizaje vinculadas a los diferentes grupos étnicos. Esto hace que, independientemente de la etnia de los hombres que habitan la casa, todos los techos metálicos sean idénticos. En consecuencia, esto implica cambios trascendentales en la percepción de la diferencia étnica en el plano de la cultura material. Es decir, supone de facto una modificación en la configuración efectiva de las identidades étnicas y su articulación a través de la cultura material ya que supone la eliminación de un elemento diferenciador claramente percibido por los individuos masculinos pertenecientes a diferentes grupos étnicos.

Por otra parte, estos nuevos materiales implican también diferentes problemas, al menos desde una perspectiva occidental. En primer lugar, el uso de cubiertas metálicas rompe 
con la tendencia a ser autosuficientes en la construcción de las casas, lo que conlleva un elevado gasto para su adquisición y genera la necesidad de entrar dentro de las redes de la economía de mercado occidental (lo que a su vez tiene un efecto cascada influyendo, por ejemplo, en los procesos de comercialización de las tierras comunales que, como comentamos anteriormente, son clave en los conflictos inter-étnicos presentes en la zona). Además, los nuevos techos aíslan muy mal, tanto el frío como el calor. Por otra parte, la adopción de plantas rectangulares que viene pareja a estos techos implica una mayor debilidad de las estructuras, en especial aquellas realizadas de tapial (Prussin 1969).

Entonces, la pregunta que surge ante todas estas desventajas aparentes es: ¿por qué adoptan los individuos estos nuevos materiales? La respuesta obtenida, tanto por nosotros como por otros autores (Drucker-Brown 2001), ha sido siempre la misma: los tejados metálicos son mejores que los tradicionales ya que implican una menor inversión de trabajo en el mantenimiento de las habitaciones (¿y por tanto más tiempo disponible para participar en las redes de economía capitalista?). Hay que tener en cuenta que las habitaciones tradicionales realizadas en adobe o tapial y con techo vegetal sufren muchos problemas de conservación durante parte del año (junio-julio y, especialmente septiembre-octubre) a causa de las fuertes lluvias. Este aspecto se ve atenuado por los nuevos techos. Los informantes reconocen los problemas de estas nuevas tipologías de habitaciones (especialmente las referidas al mal aislamiento del frío y el calor y su elevado coste), pero aun así las siguen prefiriendo.

Por otra parte, la adopción de estos nuevos techos metálicos puede ser interpretada como una nueva manera de utilizar la cultura material en la exhibición de la posición económica y política de aquellas viviendas que los utilizan. Un elemento que refuerza esta interpretación es la afirmación de Dawson (2000: 91), quien señala que normalmente son los miembros con mayor poder económico y político de los grupos konkomba quienes poseen este tipo de habitaciones. Este aspecto también lo hemos podido identificar durante nuestro trabajo de campo, añadiendo que en muchos de los compounds estudiados, estos nuevos materiales únicamente aparecen en las habitaciones adscritas al landlord. Este ejemplo ofrece una muestra de la fluidez y la contingencia que caracterizan tanto a las identidades étnicas como a la relación que éstas tienen con la cultura material. Además, muestra cómo la importancia creciente de otras identidades sociales, como es el estatus económico y político (dentro del Estado moderno ghanés y las redes de economía capitalista), puede afectar de forma directa

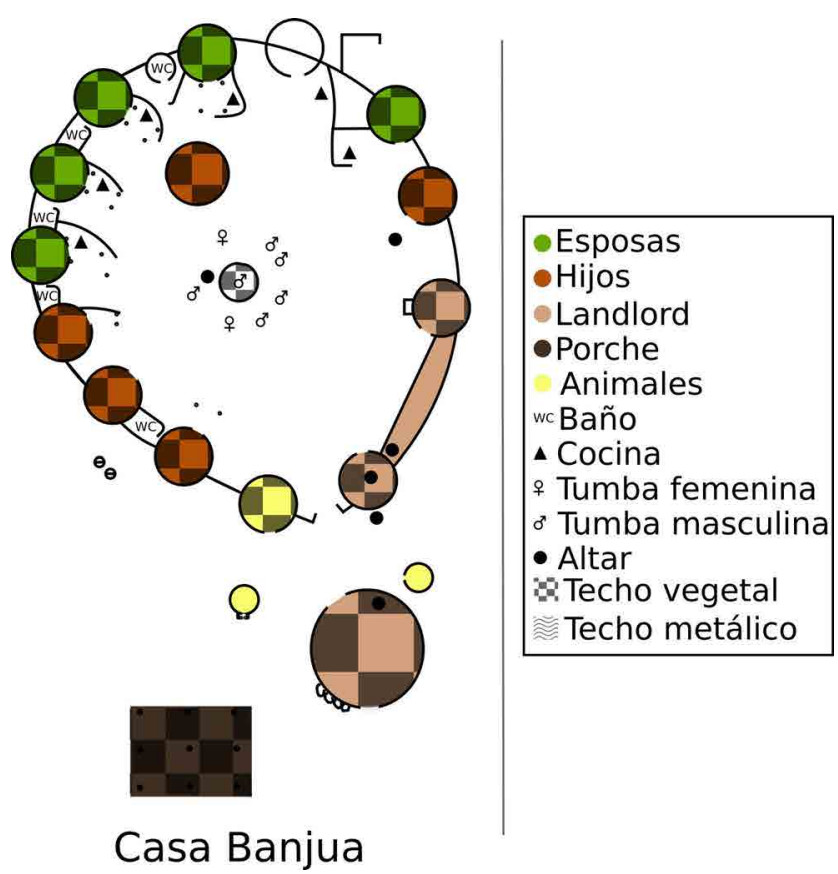

Figura 10. Planos de la Casa Banjua y la Casa Dama (etnia mamprusi, en Bende). 

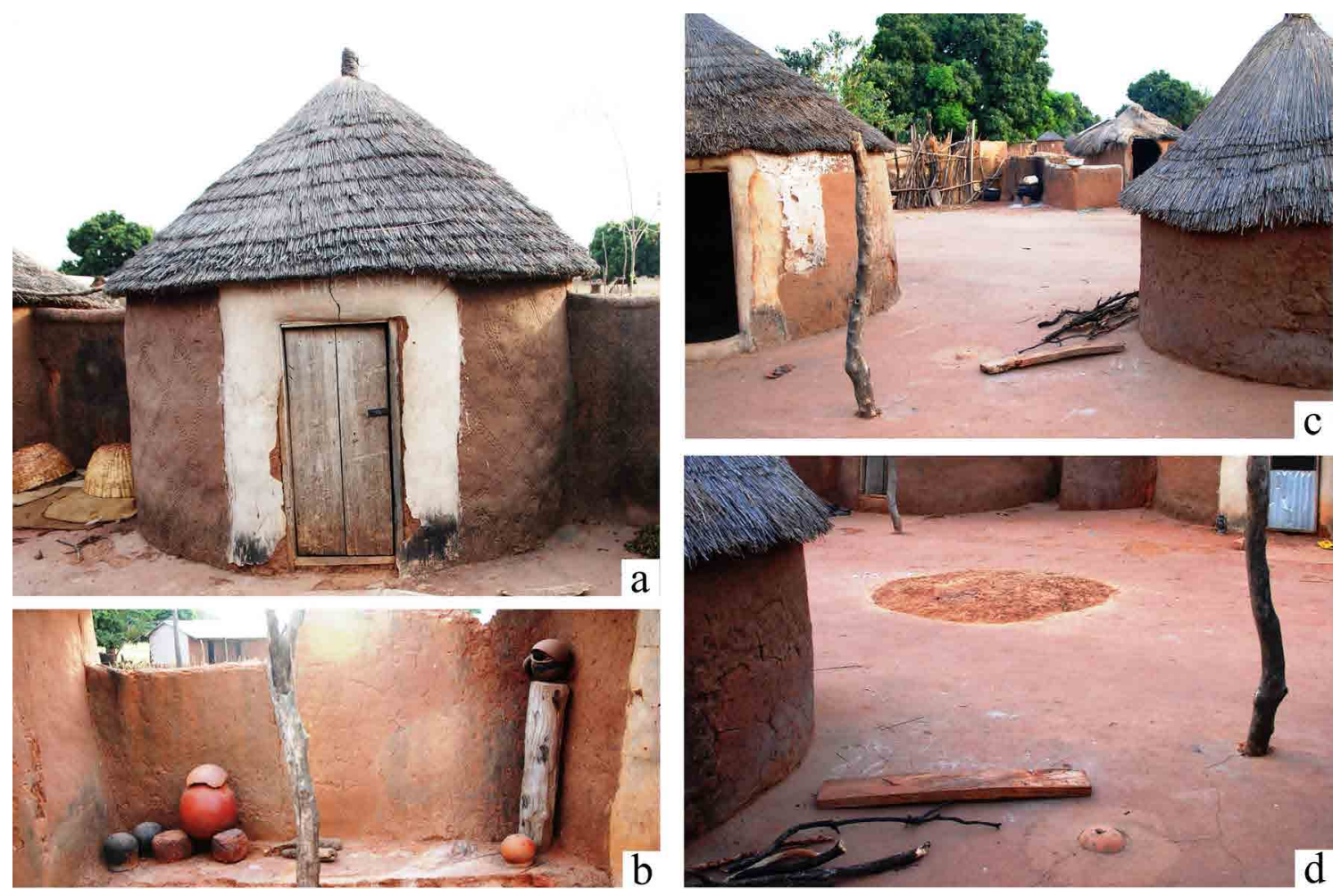

Figura 11. Casa Banjua (etnia mamprusi, en Bende). a- Habitación; b- Altares tradicionales; c- Vista general del patio central; d- Tumbas tradicionales en el interior del patio.

a la relación que se establece entre las identidades étnicas y la cultura material en los espacios domésticos.

Finalmente, y sin dejar de lado la cuestión de los nuevos materiales y tecnologías constructivas, podemos ofrecer un último ejemplo que matiza la relación establecida entre identidades étnicas y techos.

En anteriores trabajos (Calvo et al. 2013) abordamos brevemente el análisis de la casa Banjua, la casa originaria del linaje Banjua, linaje del actual jefe tradicional del poblado mamprusi de Bende. La característica más saliente de este espacio doméstico es la ausencia total de elementos constructivos "modernos", a pesar de pertenecer a un linaje con un evidente poder económico. Desde nuestro punto de vista, en la casa Banjua (Figs. 10 y 11) la exhibición del prestigio se expresa mediante una clara vinculación con el pasado y con los ancestros (que representa una de las principales fuentes de justificación del poder político tradicional de la comunidad en el difícil contexto multiétnico en el que se hallan). Este vínculo, se establece mediante dos estrategias concretas. En primer lugar, utilizando de forma exclusiva materiales y técnicas tradicionales en la construcción de la casa. Segundo, usando de forma conspicua elementos pertenecientes a la religión tradicional animista (en forma de numerosas tumbas situadas en el patio de la casa y de diferentes altares dedicados a los ancestros).

De esta manera, en este ejemplo concreto, la arquitectura tradicional, incluyendo los techos vegetales, se ha activado. Se ha convertido en un marcador étnico activo. Es decir, en este ejemplo, se ha producido una serie de decisiones y elecciones tecnológicas que han llevado a excluir los materiales y técnicas modernas primando aquellos elementos tradicionales. En definitiva, desde nuestro punto de vista, estas decisiones tienen dos objetivos. Primero, reivindicar la importancia del poder tradicional, estrechamente ligado a la identidad étnica. Segundo, enfatizar la primacía que el linaje Banjua tiene en la detentación de este poder mediante la puesta en escena de la casa principal de linaje como baluarte y refugio de la pureza mamprusi. 


\section{Conclusiones}

A lo largo de este artículo hemos pretendido mostrar como en las casas estudiadas se concentran, articulan e interrelacionan numerosas prácticas sociales esenciales en la configuración de las identidades étnicas de diversas comunidades komba, b'moba y mamprusi que habitan en el distrito de Bunkpurugu-Yunyoo, un territorio multiétnico en el que la etnicidad tiene una destacada relevancia en el día a día de estas comunidades.

Entre los elementos materiales identificados en estos espacios domésticos hemos destacado las techumbres, debido a que presentan una asociación evidente con la etnicidad. Por otra parte, los techos son uno de los elementos más conspicuos y visibles desde el exterior y tanto su fabricación como su significado se asocian de forma exclusiva con el mundo masculino. En este sentido, hemos defendido que pueden ser considerados como marcadores étnicos pasivos a través de los cuales los diferentes individuos de estas comunidades pueden identificar a qué etnia pertenecen los grupos familiares que las habitan.

Sin embargo, también hemos defendido que no debemos caer en la tentación de cosificar este hecho, pues el carácter ideacional, fluido y relacional con el que concebimos la identidad étnica se observa también en las interacciones que tienen lugar entre las personas y la propia cultura material. La identidad étnica, si bien es un fenómeno ideacional, también se concreta y se construye a partir de la cultura material.

En este sentido, en la primera parte del caso de estudio hemos analizado cómo la construcción de las techumbres y su visualización permite una identificación de las diferencias étnicas en tanto que son el proceso de habitus tecnológicos concretos transferidos a lo largo del tiempo a través de redes de aprendizaje que se mueven por la vía masculina, que es la que también articula la afiliación étnica de las comunidades. De esta manera, se explica que cada grupo étnico tenga características diferenciadas a la hora de fabricar estos elementos.

Por otro lado, ya hemos dicho que no concebimos las identidades sociales como entidades fijas e inmóviles, sino como procesos (Gosselain 2000: 188). Es decir, defendemos que son contextuales, multiformes, complejas, contradictorias y están en perpetuo cambio. En consecuencia, argumentamos que la cultura material que se inserta en sus dinámicas comparte estas mismas características. En este sentido, a través de cuatro matices concretos hemos tratado de concretar esta idea, que nos permite avanzar en el conocimiento de las complejas relaciones existentes entre cultura material e identidades étnicas.

En primer lugar, hemos enfatizado el carácter relacional de las identidades étnicas $y$, por tanto, de la cultura material asociada a ellas. En este sentido, hemos señalado que la relación existente entre tipos de techos e identidades étnicas únicamente se observa en el área de estudio. Una de las principales razones para que esto ocurra debe buscarse en la realidad multiétnica existente en el territorio que, como hemos visto, supone la existencia de complejos conflictos que afectan al día a día de las comunidades que viven en el distrito de Bunkpurugu-Yunyoo. En definitiva, defendemos que las filiaciones étnicas en este territorio tienen una mayor relevancia que en otras zonas donde existe una mayor homogeneidad étnica. Esta realidad debe relacionarse con la propia estructura política de Ghana y con el acceso al poder político (y por tanto al derecho de la propiedad de la tierra) que tienen cada uno de los grupos étnicos en el territorio estudiado. Por todo ello, la filiación étnica y su relación con algunos aspectos de la cultura material es especialmente visible en este distrito.

Así como las identidades étnicas son relacionales, es decir, se activan especialmente en relación al "otro" la cultura material que funciona inmersa en estas dinámicas también se muestra especialmente activa en relación a los identificadores étnicos pasivos que cada grupo articula dentro de su propia cultura material. Por ello, al contrario de lo que ocurre en este distrito en otras zonas con mayor homogeneidad étnica (o con otros grupos étnicos en conflicto) los techos adquieren este carácter identitario que hemos analizado.

Si este primer ejemplo nos ha permitido ver el carácter relacional de la cultura material con el segundo y el tercer ejemplo hemos pretendido enfatizar el carácter fluido de la identidad y de la propia cultura material que intersecciona con ella. Este carácter fluido debe ser entendido en dos de sus posibles acepciones: la primera que evidencia como la identidad étnica se construye de manera distinta entre hombres y mujeres (segundo ejemplo) y la segunda como la identidad que se activa o desactiva contingentemente en función de los intereses de los grupos o de las personas (tercer ejemplo). 
Como hemos visto, la techumbre, visualizada desde el exterior identifica en un primer momento la afiliación étnica de los habitantes de la casa, aunque una vez en el interior, y en especial, con la cultura material asociada a la mujer que no tenga la filiación de su marido (decoraciones y uso de cerámica), pone en evidencia otras identidades étnicas distintas a la del landlord.

Estas dinámicas enfatizan la plasticidad de las identidades y otorgan agencia a los actores sociales que pueden, a través de la cultura material, resaltar y/o modificar sus múltiples identidades en función del contexto y sus intereses. En base a ello, Casella y Fowler (2004: 2) defienden que las identidades son experiencias transitorias a nivel sincrónico. Es decir, éstas son siempre múltiples y se van articulando en un momento y en un espacio determinado. Ejemplo de ello sería la confluencia de diferentes identidades sociales y distintas filiaciones étnicas en una misma casa y su distinta dinámica con la cultura material. A su vez, estos mismos autores argumentan que las identidades son transitorias también a nivel diacrónico, en el sentido de que van cambiando a lo largo del tiempo. Los cambios entre identidad y cultura material que se generan con la introducción de las nuevas techumbres de zinc nos remiten a esa transitoriedad diacrónica desde una doble perspectiva: activación de nuevas identidades sociales relacionadas con el poder adquisitivo y ruptura de la relación como mar- cador identitario étnico que existía entre la techumbre de la casa y la filiación del landlord.

Finalmente, también hemos visto cómo la cultura material implicada en los procesos de conformación, mantenimiento y gestión de identidades étnicas también comparte el carácter ideacional de éstas. En primer lugar, este fenómeno se documenta a partir del reconocimiento, por parte de los individuos de estas comunidades, de las diferencias existentes entre las diferentes maneras de construir los techos. Estos pasan a ser identificadores étnicos pasivos única y exclusivamente porque son percibidos como diferentes por los individuos, y esa diferencia les permite asociarlos a un grupo étnico. En segundo lugar, con el ejemplo de la casa Banjua hemos visto como también, en algunos casos concretos, esta misma materialidad se convierte en un identificador étnico activo con un carácter marcadamente ideacional. Este fenómeno ocurre cuando la cultura material es utilizada conscientemente para visualizar un determinado tipo de identidad étnica. En este sentido, toda la materialidad de la vivienda de la familia Banjua, supone un ejercicio activo, no solo por mantener la cultura material tradicional asociada a las casas, sino que también, con su ejemplo, la casa y por ende la familia que la habita, se referencian como garantes del mantenimiento de las tradiciones, de la misma manera que el cabeza de dicha familia, el jefe Banjua II, lo es para la comunidad mamprusi del poblado de Bende.

\section{Notas}

3. Konkomba es el término más utilizado para referirse a este grupo étnico en la literatura antropológica, aunque ellos se refieren a sí mismos como Bekpokpam. Dawson $(2000,2009)$ afirma que los konkomba de Ghana se dividen en dos grupos diferentes, los komba en el norte y los bimotiev en el sur. En este trabajo nos referimos principalmente al grupo del norte, a los komba, aunque en algunos casos nos referiremos de forma general a ambos grupos y utilizaremos el término konkomba.

4. En el territorio analizado habitan también individuos y familias pertenecientes a los grupos étnicos fulani, talensi, moshi, hausa, dagomba, chokosi o busanga. Sin embargo, su número en la zona es muy reducido $\mathrm{y}$ no afectan a las cuestiones tratadas en este trabajo.

5. Esto parece ser cierto en el territorio aquí analizado, en el momento en el que realizamos nuestro trabajo de campo. Sin embargo, hemos documentado habitaciones circulares con techos de zinc en otras zonas del NE de Ghana.

\section{Bibliografía}

Barth, F. (1969): Ethnic groups and boundaries: the social organization of culture difference. Little, Brown, Boston. 
Bentley, G.C. (1987): Ethnicity and practice. Journal of comparative study of society and history, 29: 24-55.

Bourdieu, P. (1970): La Maison Kabyle ou le monde renversé. Echanges et communication. Melanges offerts a Claude Levi-Strauss (J. Pouillon, P. Maranda, eds.), Mouton, The Hague: 739-758.

Bourdieu, P. (1977): Outline of a Theory of Practice. Cambridge University Press, Cambridge.

Bourdieu, P. (1990): The logic of practice. Stanford University Press, Stanford.

Calvo, M.; Fornés, J.; García Rosselló, J.; Javaloyas, D.; Sastre, M. (2013): Houses and society. An ethnoarchaeological study of the mamprusi's houses (Bende, Northeast Ghana). Ethnoarchaeology: current research and field methods (F. Lugli, A.A. Stopiello, S. Biagetti, eds.). Archaeopress, Oxford: 31-35.

Calvo, M.; Albero, D.; García Rosselló, J.; Javaloyas, D.; Gavua, K.; Fornés, J. (2016): I Like You, I Like Your Pottery: An Ethnoarchaeological Approach to Ceramic Distribution and Acquisition in Northeastern Ghana. African of Archaeological Review, 33(3): 297-320. http://dx.doi.org/10.1007/ s10437-016-9225-Casella, E.; Fowler, C. (Eds.) (2004): The Archaeology of Plural and Changing Identities: Beyond Identification. Kluwer Academic Publishers Group, Dordrecht.

Curta, F. (2014): Ethnic identity and archaeology. Encyclopedia of Global Archaeology (C. Smith, ed.), Springer Reference, New York: 2507-2514.

Dawson, A.C. (2000): Becoming Konkomba: Recent Transformations in a Gur Society of Northern Ghana, (unpublished thesis). Department of Anthropology University of Calgary, Alberta.

Drucker-Brown, S. (2001): House and hierarchy: politics and domestic space in Northern Ghana. Journal of Royal Anthropological Institute, 7: 669-685.

Emberling, G. (1997): Ethnicity in complex societies: anthropological perspectives. Journal of Archaeological Research, 5: 295-344.

Eriksen, T. H. (1993): Ethnicity and nationalism. Anthropological perspectives. Pluto Press, Londres.

García Rosselló, J.; Javaloyas, D.; Albero, D.; Calvo, M. (2012): Palabras que no se lleva el viento. La conciencia histórica de las comunidades mamprusi del clan Sala (NE Ghana). Oráfrica, Revista de Oralidad Africana, 8.

González-Ruibal, A. (2003): La experiencia del otro. Una introducción a la etnoarqueología. Akal, Madrid.

González-Ruibal, A. (2006): Order in a disordered world. The bertha house (Western Ethiopia). Anthropos, 101: 379-402.

Gosselain, O. (2000): Materializing Identities: An African Perspective. Journal of Archaeological Method and Theory, 7 (3): 187-217. https://doi.org/10.1023/A:1026558503986

Hall, J. (1997): Ethnic identity in Greek antiquity. Cambridge University Press, Cambridge.

Hodder, I. (1982): Symbols in action. Cambridge University Press, Cambridge.

Hodder, I. (2012): Entangled: an archaeology of the relationships between humans and things. Wiley Blackwell ed. Chichester, West Sussex.

Jones, S. (1997): The archaeology of ethnicity: constructing identities in the past and present. Routledge, London.

Knapp, B. (2001): Archaeology and ethnicity: a dangerous liaison. Archaeologia Cypria, 4: 29-46.

Latour, B. (2008): Reensamblar lo social: una introducción a la teoría del actor-red. Ed. Manantial, Buenos Aires.

Lewis, M. P. (ed.) (2009): Ethnologue: Languages of the World, Sixteenth edition. SIL International. Online version, Dallas.

Meskell, L. (2001): Archaeologies of identity. Archaeological Theory: Breaking the Boundaries (I. Hodder, ed.), Polity Press, Cambridge: 187-213.

Meskell, L. (2002): The intersections of identity and politics in archaeology. Annual Review of Anthropology, 31: 279-301. https://doi.org/10.1146/annurev.anthro.31.040402.085457

Olsen, B. (2003): Material culture after text: re-membering things. Norwegian Archaeological Review, 36 (2): 87-104. https://doi.org/10.1080/00293650310000650

Olsen, B. 2010. In Defense of Things. Altamira Press, Walnut Creek.

Owusu-Mensah, I. (2014): Politics, Chieftaincy and Customary Law in Ghana's Fourth Republic. The Journal of Pan African studies, 6 (7): 261-278.

Prussin, L. (1969): Architecture in northern Ghana: a study offorms and functions. University of California Press, Berkeley.

Reher, G. S. (2011): The 'Introduction to Ethnicity Syndrome' in Proto-historical Archaeology. Atlantic Europe in the First Millennium BC: Crossing the divide (T. Moore; X.L. Armada, eds.). Oxford University Press, Oxford: 656-667.

Renfrew, C. (1987): Archaeology and language: the puzzle of Indo-European origins. Jonathan Cape, London.

Shennan, S. (1989): Introduction: archaeological approaches to cultural identity. Archaeological approaches to cultural identity (S. Sherratt, ed.), Routledge, London: 1-32. 
Sweeney, N. (2009): Beyond Ethnicity: The Overlooked Diversity of Group Identities. Journal of Mediterranean Archaeology, 22 (1): 101-126. http://doi.org/b9fhxv

Smith, A.D. (1986): The ethnic origins of nations. Basil Blackwell, Oxford.

Smith, M. (2007): Inconspicuous Consumption: Non-Display Goods and Identity Formation. Journal of Archaeological Method and Theory, 14 (4): 416-417. https://doi.org/10.1007/s10816-007-9040-6

Trigger, B. G. (1989): A History of Archaeological Thought. Cambridge University Press, Cambridge.

Weber, M. (1968) [1922]: Economy and Society. Bedminster, New York.

Yaeger, J.; Canuto, M. (2000): Introducing an archaeology of communities. The Archaeology of Communities: A New World Perspective (M. Canuto; J. Yaeger, eds.), Routledge, London. 